\title{
SIMULTANEIDAD Y COMPLEMENTARIEDAD ENTRE ECONOMÍA Y EDUCACIÓN
}

ISSN 2219-6722

ISSNE 2222-2707

\begin{abstract}
JORGE FLORES SILVA, Instituto de Investigaciones Económicas y Sociales
\end{abstract} Universidad Nacional Autónoma de Honduras (UNAH)

\section{RESUMEN}

Con las exigencias de la economía global, se impulsan modelos educativos para la eficiencia y el mercado. Todo se mercantiliza hasta el conocimiento y se da primacía a la educación Tecnocrática que privilegia lo virtual sobre lo real, corriendo el riesgo que lo técnico opaque el conocimiento y la investigación que lleva al descubrimiento.

Como lo que se busca es la rentabilidad económica en función del interés privado, entonces el estudio de la filosofía, la historia y la economía política, entre otras disciplinas se descuidan o se abordan de manera marginal sobre todo en las universidades Privadas.

La educación es un reflejo del modelo económico que se impulsa, la política educativa queda subordinada a la política económica y la actividad educativa se incorpora bajo la modalidad de las actividades simples y mecánicas que desarrolla un aparato productivo rezagado. Un sistema educativo tecnocrático y un aparato productivo eficientista toma como punto de referencia estas acciones simples, bajo la perspectiva de habilidades técnico-motrices, como elementos de evaluación y desempeño de los graduados y egresados de las instituciones educativas en los tres niveles. De esta manera se reduce la educación a una esfera de la personalidad humana relacionada con actividades motoras. No se considera la dimensión integral de la educación que prepara a los educandos para la vida y su interrelación en el contexto del cual forma parte.

Palabras clave: economía, educación, sujeto, tecnología, productividad. 


\section{SIMULTANEITY AND COMPLEMENTARITY BETWEEN ECONOMY AND EDUCATION}

ISSN 2219-6722

ISSNE 2222-2707

JORGE FLORES SILVA, Instituto de Investigaciones Económicas y Sociales

Universidad Nacional Autónoma de Honduras (UNAH)

\section{SUMMARY}

With the demands of the global economy, educational models for efficiency and drive the market. Everything is commodified to knowledge and gives primacy to the Technocratic education that favors the virtual over the real, the risk that opaque technical knowledge and research leading to the discovery.

As what is sought is the economic profitability based on private interest, then the study of philosophy, history and political economy, among other disciplines are neglected or marginally addressed especially in private universities.

Education is a reflection of the economic model that is driven, educational policy is subordinated to economic policy and educational activity is incorporated in the form of simple and mechanical activities developed a lagging productive apparatus. A technocratic education system and an efficiency productive apparatus takes as a reference point these simple actions, from the perspective of technical- motor skills, as elements of evaluation and performance of graduates and graduates of educational institutions at all three levels. Thus education is reduced to a sphere of human personality related to motor activities. It is not considered integral dimension of education that prepares students for life and their interrelation in the context of which it is part.

Keywords: economy, education, subject, technology, productivity. 


\section{INTRODUCCIÓN}

La ciencia y el conocimiento que implica debe democratizarse, pero antes deben impulsarse cambios objetivos que mejoren las condiciones materiales de existencia de la gente, para que esta misma gente tenga acceso a los bienes de la educación como derecho humano, y que al estudiante no se le vea como un cliente más del proceso educativo. Esto también implica cambios subjetivos, orientados al impulso de una educación sobre la base de un curriculum integral, que verdaderamente humanice y socialice para el deber ser. Reconociendo al ser humano como persona que posee dignidad, inteligencia, libertad y voluntad.

En una concepción científica de la educación, el sujeto en quien recae la acción educativa, se forma para tener una visión crítica del mundo y del contexto socio-económico que le rodea, solo con una educación que socializa y humaniza, que lo hace comprender que debemos afirmarnos como sujeto luchando por una economía para la vida y una enseñanza del deber ser se adquirirá una conciencia crítica para emprender la acción transformadora en un mundo complejo, dinámico y lleno de incertidumbre.

La acción social, implica una acción económica y tiene implicación política. El teólogo Leonardo Boff afirma la política en el buen sentido de la palabra "...es educación, es salud, es inclusión, es empleo, es cuidado de la naturaleza..." Los resultados de la acción educativa y los problemas de pobreza que en nuestro país tienen un carácter estructural, son temas que en la actualidad no pueden apartarse de la agenda nacional. Se han invertido grandes cantidades de recursos tanto en la educación, como en el combate de la pobreza, y la pobreza y el problema del empleo que están íntimamente relacionados siguen en aumento. La planificación ha sido puramente formal, los objetivos estratégicos que se formularon en los planes de desarrollo del pasado, todavía tienen vigencia.

En tal sentido será necesario considerar para cualquier disciplina la dimensión económica que permita, desde luego, articular la demanda socio-productiva, con la oferta profesional, no pensando únicamente en la eficiencia económica en función del interés particular que excluye a los más débiles y a la colectividad.

En un mundo materialista donde priva la acumulación y una economía utilitarista para la eficiencia y la máxima ganancia, adquirir conocimiento quizá no interese, sobre todo en países pobres donde prevalecen los procesos simples y se da prioridad casi y exclusivamente a la adquisición de habilidades motrices en la preparación del ser humano para formar capital. Todo se ve como capital, todo es reemplazable y se tiende a acabar con todo de manera desmedida. Al ser humano con ciertas destrezas, habilidades y mínimos conocimientos se le caracteriza como capital humano. En este concepto se encierra una dimensión ética, porque el capital se desprecia, por el desgaste que sufre ejemplo la maquina como bien de capital, se deprecia el dinero como capital financiero. Así entonces el ser humano, desde esta perspectiva también se deprecia cuando deja de ir a la escuela, cuando se envejece o cuando se le olvidan las cosas. A este grado se ha llegado en una sociedad donde poco a poco se ha ido perdiendo la noción de lo que es bueno y de lo que es malo. El ser humano no puede ser capital, el ser humano es ante todo persona que está dotado de inteligencia, voluntad y libertad. Que tiene dignidad independientemente de su condición social, económica, raza o país de origen de donde proviene.

\section{La educación y la economía como práctica científica que implican significados}

La educación y la economía como ciencias ideográficas deben tratarse de manera simultánea y complementaria, basada en el conocimiento del contexto histórico y de la realidad observable, que no solo permita describir, sino al mismo tiempo cuestionar mediante el análisis y visión crítica para una acción transformadora.

Lo anterior implica, no solo encerrarse en una sola línea de pensamiento, sino en comunión de ideas 
y pareceres adoptar una posición ecléctica para el diseño, construcción y aplicación de un curriculum educativo que rompa con el mito de que con solo educación se va a salir de la pobreza. Si no hay una economía con dimensión ética, difícilmente se podrá salir del círculo vicioso de caída de la demanda, baja inversión, desempleo, caída de los ingresos, pobreza...

La educación constituye una práctica social, para el deber ser, que forma al individuo de manera integral que tiene que ver con toda la esfera de la personalidad, la esfera psico-bio-social. Por tanto, la educación que humaniza y socializa no puede formar personas para que se les califique como capital. Si así fuera el proceso enseñanza-aprendizaje seria deshumanizante. Es inconcebible hablar de educación en valores, porque la educación implica de por si educar en valores y si no fuera así, dejaría de ser educación.

Lo mismo pasa en la perspectiva ecológica. "Los procesos en la medida que P. Richard "son excluyentes de las mayorías y destructores de la naturaleza, tienen una cultura, una ética y una espiritualidad más de muerte que de vida" (P. 228). La economía ambiental, como la economía verde, aunque sus principales exponentes abundan en consideraciones que parecieran que están preocupados por el cuidado, rehabilitación y conservación de la naturaleza, pero son simples considerandos. Es en la economía ambiental, donde se generan conceptos como el de capital natural y capital artificial. Esta perspectiva trata de acabar con todo lo que existe, donde priva el abuso y no el uso de los recursos naturales. W. Dierckxsens 2009 "No tiene sentido desarrollar más capital artificial cuando el capital natural ya no puede reponerse”. Es la economía ecológica que estudia y plantea soluciones para el buen uso de los recursos extraídos de la naturaleza, si se quiere garantizar que existan bienes, trabajo y consumo para la existencia de la humanidad, que presupone la existencia de la naturaleza. En tal sentido las prácticas de trabajo y los estilos de vida, deben estar orientados para vivir y explotar recursos de manera racional que a su vez garantice que se vive en igualdad de condiciones con la naturaleza -fuente de vida y riqueza para la humanidad-.

Hasta aquí lo tratado no es ajeno al campo de la economía de la educación, es esta disciplina que invita y explica las particularidades y peculiaridades de la gente, como también explica los desequilibrios de la oferta laboral con la demanda laboral. El ser humano como fuente de riqueza, la naturaleza y el capital, constituyen el motor para el aumento de la base material de la sociedad. Es sobre todo el ser humano con el equipo y herramientas que ejerce su acción sobre la naturaleza para aumentar la capacidad productiva de la sociedad. Este aumento es mayor en la medida que al ser humano se le capacita y se le educa de manera continua y permanente para ir alcanzando estadios de desarrollo en donde los sectores de la economía logren su intersectorialidad. De manera que la población desplazada como incremento del capital y la productividad pueda ser absorbido por los demás sectores productivos. Así el sector agrícola al desplazar mano de obra, este es absorbido por el sector industrial y el que sea desplazado del sector industrial como resultado del proceso de desarrollo pueda ser absorbido por el sector servicios. Este proceso refleja la relación del nivel educativo con el sector donde se opera, de modo que es fácil ver el nexo existente entre nivel primario con el sector agrícola, el nivel educativo secundario con el sector industrial y el nivel educativo superior con el sector servicios. Esta relación, educación-trabajo debe mover a la institución educativa, sobre todo a la del nivel medio y superior a garantizar nuevos procesos, nuevos métodos para nuevas actividades laborales, con ajuste del curriculum educativo y la innovación tecnológica.

Otro fenómeno que vale la pena abordar desde la perspectiva del capital humano es: la obsolescencia temprana de los bienes. Si los bienes tienen una vida útil corta implica que el ser humano debe estar adquiriendo nuevos conocimientos para su uso, manejo y estar a tono con las nuevas adquisiciones. Si continuamente surgen nuevos aparatos con nuevas tecnologías, entonces a ese mismo ritmo se reduce 
el uso y valor de las existentes y si las personas que la utilizan o los trabajadores que los manipularan no se forman, caerán al mismo ritmo en obsolescencia temprana en el campo de la educación y la adquisición de habilidades. Por eso debe haber educación en el trabajo, formación continua en las instituciones educativas y preocupación de las personas por continuar estudiando toda su vida.

En relación a lo anterior y como parte de la educación para la economía, parafraseando a W. Dierckxsens "la planeación de la obsolescencia temprana de los bienes desde la óptica de hacer dinero genera riqueza, pero desde el punto de vista del contenido genera pobreza porque es de la naturaleza de donde se saca la materia prima para la producción de tales bienes. Si la vida media de los bienes se reduce, entonces la vida media de la naturaleza también disminuye y por consiguiente la vida media del ser humano tendera a disminuir. Porque la sobrevivencia del ser humano depende de la naturaleza." El rezago del aparato productivo, la práctica de procesos y actividades simples, la poca investigación en terreno, son causa para que el sector productivo y de servicios no se interese por la contratación de personal calificado. Da lo mismo contratar un analfabeto, de poca escolaridad o medianamente calificado para que sus objetivos sobre los cuales centra su visión se realicen. Más aun en una economía caracterizada por desequilibrios entre oferta laboral y demanda laboral, quienes tienen los medios de producción son los que tienen la opción de invertir y al haber sobre abundancia de personal calificado y no calificado desempleado, quien invierte juega con los salarios. Esto no solo genera desinterés por incorporar progreso técnico, porque prefiere contratar mano de obra abundante en desempleo para pagarle inclusive hasta por debajo del salario mínimo. En el "mejor de los casos" para el empleador, es contratar personal de buen nivel académico, inclusive hasta universitario aprovechándose de la sobreabundancia de mano de obra y así pagarle apenas el salario mínimo. De aquí surge la famosa frase "pobres ilustrados" (R. Perdomo)

El rezago que padece nuestra economía y las unidades de producción enlazadas en el aparato productivo, no son imputables con exclusividad a la falta de personal calificado, ni tampoco puede atribuírsele solo a los egresados y a las entidades educativas con todos sus actores la falta de oportunidades para enrolarse en un trabajo que le permita vivir con dignidad, como a veces se pretende hacer creer cuando se afirma que los titulados de los centros educativos no encuentran empleo porque salen mal preparados, esto no es del todo cierto o es una visión de la realidad sin o con poco fundamento, porque a pesar de las debilidades del sistema educativo que son muchas, lo real es que en alguna medida la propia dinámica del mercado laboral, los patrones de apropiación y explotación de los factores de producción, la estructura de consumo y el carácter de la incorporación e innovación tecnológica, son entre otros factores los causantes para que una gran cantidad de jóvenes bien preparados no sean absorbidos por el sistema.

\section{La educación es un derecho, pero también es una inversión}

Como derecho, debe ser prioridad en la agenda de los gobiernos y de la cooperación internacional. Evitando hacer de ella un medio para la máxima ganancia en función del interés privado. Sobre todo, la educación de los primeros niveles debe procurarse y garantizarse su gratuidad y de buena calidad, considerando que, en la externalidad, no solo gana quien recibe la educación, sino la sociedad toda. Pero la educación también es una inversión, que genera rendimientos a quien la recibe. Rendimientos tangibles que se expresa en los ingresos que recibe el individuo educado y capacitado, pero más importante es el rendimiento que no se expresa monetariamente. La persona preparada obtiene un potencial de ingresos que puede ir en aumento según los años de educación, esta situación le facilita entrar en relaciones de mercado, por el poder de compra que puede facilitarle en una economía de mercado ascender en la escala social.

La relación de eficiencia positiva en el proceso de trabajo que desarrolla la persona formada, aumenta 
la producción y disminuye costos en la unidad económica donde labora, sea propia o no. Entiende y se le facilita dar y recibir instrucciones, administra mejor el tiempo con el método de reducir tiempos y movimientos, emplea de manera más eficiente los recursos disponibles incluyendo la habilidad para evitar desperdicios. Estas y otras ventajas que da la capacitación formal y no formal, es lo que se puede resumir en aumento de la PRODUCTIVIDAD.

Este incremento de la productividad de los factores de producción varia de una sociedad a otra, así la productividad del trabajo es mayor en las sociedades desarrolladas, por la capacidad de asimilar las nuevas tecnologías que en esa misma sociedad se genera, en virtud de que también su gente ha recibido mayor educación. La productividad del capital también es mayor, casi por las mismas razones. Así una sociedad tradicional emplea más tierra que capital, en tanto que una comunidad más avanzada tecnológicamente tiende a emplear más capital y con el mismo o menor espacio físico obtener mayores rendimientos. De esta manera también se refleja la productividad de la tierra que se incrementa por innovadoras prácticas de cultivo, por la incorporación del progreso técnico y por las habilidades y destrezas de quienes realizan el trabajo. En todo ello tiene que ver la educación y consecuentemente genera mayores ingresos a los protagonistas del proceso que siempre son los seres humanos, como auténticos generadores de riqueza.

Aunque el mito de que con solo educación se va a salir de la pobreza, se viene abajo en algunos países, como Honduras que tienen mucho personal altamente calificado que vive en la pobreza, esto es así porque la mayoría de los empleadores que los invade el "miedo" a emprender procesos complejos y con ello a no emplear personal calificado a quienes tendrían que ofrecerles mayor remuneración por su trabajo. También es cierto que se encuentran casos eventuales de personas que obtienen altos rendimientos en las actividades que realizan, algunas de las cuales están dentro del sector informal de la economía, sin ser personas de mayor educación.

Esta situación permite concluir que educación y economía son procesos y prácticas que implican significados que deben verse de manera simultánea y complementaria, ante la disyuntiva de estudiar o trabajar para ayudar a la manutención personal o familiar. O estudiar y sin tener la posibilidad de acceder al ingreso que permita contribuir al sustento de la familia sobre todo en aquellos hogares pobres.

De todos modos, hay que reconocer que hay más posibilidades de salir de la pobreza en la medida que se tenga mayor escolaridad, existiendo en la generalidad de los casos una relación directa entre educación y ganancias así: mayor educación mayores ingresos, o relación inversa: menor educación mayor pobreza. De aquí surge la relación entre el nivel educativo y el sector económico que asocia nivel educativo primario con sector primario de la economía (la agricultura); nivel educativo de la secundaria con el nivel secundario de la economía (industria) y nivel educativo terciario o superior universitario con el nivel terciario de la economía o de servicios ejemplo el sistema financiero.

Tener una visión tecnocrática de la educación, y una noción de la economía solo para el cálculo de la utilidad alejándola de su orientación para la vida son prácticas que deben superarse, mediante la postulación de ideas y creación de ciencia que permita replanteamiento de alternativas para el desarrollo en función del desarrollo de la persona.

\section{Motivaciones por el deseo de saber}

Nadie es absolutamente ignorante, cada quien es profesional en su área de desempeño, la enseñanza que nos da tanto la escuela de la vida, como la enseñanza como procesos continuos en las actividades cotidianas para lograrse el sustento. Así como un buen abogado quizá no sepa sembrar una milpa o levantar una pared, sin embargo, puede ser un brillante juez que sabe interpretar bien lo que es la justicia y el derecho... 
Todos tenemos algo que enseñar y todos tenemos algo que aprender del otro... no somos autosuficientes, saber o tener algunos conocimientos, no significa saberlo todo. Sin embargo, siempre el ser humano aspira a saber más a través de la educación sistemática o formal. La búsqueda de la verdad nos empuja el deseo de saber, porque tenemos motivaciones tanto materiales como solidarias. El ser humano busca educarse porque tiene aspiraciones monetarias o materiales, se educa para ser más competitivo, para ganar dinero y adquirir cosas o bienes tangibles que pueden hacer visible su status que utiliza como referente ante los demás. Trasluce el ego y la vanidad de la naturaleza humana. El vacío existencial muchas veces se pretende llenar con cosas. La felicidad pasajera de los bienes puede desembocar en frustración.

Solo cuando comprendemos que lo invisible es más importante que lo visible, el sentido trascendente de nuestra vida va dando paso a las motivaciones solidarias para aspirar a una educación que nos impulse a ayudar a los demás. Es aquí donde encajan las razones no monetarias de la educación. El deseo de educarse tanto para saber más como para ayudar a los demás, a sustituir una educación utilitarista y tecnocrática por una educación solidaria y del deber ser. Una educación que como práctica social implica significados, que socializa y humaniza. Una educación no solo para vivir o vivir mejor, sino una educación para saber convivir.

La educación se gane dinero o no se tenga ningún rendimiento monetario siempre genera bienestar. Estos momentos agradables de la vida estudiantil en donde se tiene la oportunidad de interactuar con compañeros de estudio y académicos, se extienden hasta la vida profesional y en sus experiencias de vida.

Compartir el proceso enseñanza-aprendizaje siempre genera satisfacción, es en la vida estudiantil donde se comparten momentos alegres propios de la juventud, se introduce a la incubadora de ciencia y surge un investigador junior en la disciplina de que se trate, se desarrolla el potencial creativo, se comparten conocimientos y experiencias reciprocas de todo el bagaje de información al cual el mundo de hoy nos permite entrar por el avance de las tecnologías de información y el cálculo rápido. El gusto del motivo intrínseco por saber nos produce bienestar que tiene un valor incalculable que no se mide monetariamente.

\section{La complementariedad entre educación y economía}

La educación no solo es una inversión económica, es también una inversión social que genera un rendimiento social, es un rendimiento no solo de corto plazo es un rendimiento que dura toda la vida, nos permite dar sentido social al conocimiento, darle significado al dato, analizar cualitativamente el pasado y el presente, postular ideas para el futuro. No solo se estudia para obtener un trabajo y recibir una remuneración en dinero, se estudia para analizar comprender la realidad de nuestro entorno y del contexto mundial del cual formamos parte. J. Malta Luna 2002 "Con inversión educativa e investigación científica los países se aproximan más al desarrollo. De manera individual, la persona instruida y educada está más preparada para afrontar la vida... también tiene más oportunidades de desenvolverse en sociedad y conseguir reconocimientos sociales, como de sentirse realizado como persona..." (P.119) Una madre educada tiene mayores posibilidades de transmitir valores a su hijo, servir de estímulo intelectual y apoyarlo en sus tareas escolares. Estos resultados le causaran placer como pedagoga natural para sus hijos, aunque no tenga rendimientos monetarios tangibles.

La educación para la vida siempre será más importante que la educación utilitarista para la ganancia. La educación utilitarista que instrumentaliza está subordinada a la economía para la máxima ganancia que excluye y destruye la naturaleza. En cambio, la educación para la vida está asociada a la economía para la vida, donde no interesa solo el rendimiento monetario o la máxima ganancia, sino que interesa la posibilidad de vivir mejor a través del acceso a los bienes culturales, a los bienes sociales y a los espirituales que permiten una felicidad más duradera. Aunque esta última perspectiva es suficiente 
para lograr el desarrollo a través del desarrollo de la persona, vale la pena destacar que resultaría doblemente mejor al postular una educación y una economía donde no haya supeditación de una sobre la otra, sino que se vieran y trataran de manera simultánea y complementaria ante la disyuntiva de trabajar o estudiar o viceversa.

El modelo educativo y el modelo de desarrollo que impera, el padre de familia o la madre soltera y con hijos se pregunta, o mando a mis hijos a la escuela y nos quedamos sin comer, o los mando a trabajar para que ayuden al sustento familiar, aunque no vayan a la escuela. Cualquiera de las dos opciones resulta en una tragedia que engendrara el círculo de la miseria y la tragedia de la desesperanza. Entonces la simultaneidad y complementariedad es importante para que haya educación y que haya economía, donde se garantice una educación como derecho humano y un empleo para los padres que les permita vivir con dignidad y de autorrealización para ellos y sus dependientes.

Esta postura, aunque parezca una utopía, puede hacerse realidad si se replantea una alternativa de desarrollo en función del desarrollo de la persona, porque hay más posibilidades de hacer desarrollo en la medida que se invierte en las personas. Esto implica juzgar y cuestionar la racionalidad del proceso económico - social que aparta a la educación y a la economía de su orientación para la vida.

Este replanteamiento solo se realizará, cuando no se juzgue el hecho educativo solo como beneficio individual de quien recibe la educación, sino que implica tener rectitud de conciencia que el beneficio individual de la educación, es también beneficio social para la sociedad en su conjunto.

El beneficio de la educación individual, hacia la sociedad, empieza por la transmisión de la cultura, los valores, el conocimiento y todos los saberes desde los padres de familia a sus hijos, de tal modo que primero se transmite en el propio hogar, y si la sociedad en sentido práctico se concibe como el conjunto de los hogares esta verdad es incuestionable e inacallable. Esta realidad presupone la existencia de hogares, pero que pasa en una sociedad donde "no hay hogares", porque el despojo el desplazamiento que sufre la familia termina en desintegración, de la primera célula o corazón de la sociedad. La respuesta que salta a la vista es que se está ante una crisis de la familia, como crisis ética que amenaza a los países.

\section{La crisis como amenaza global}

Esta crisis que se está convirtiendo en crisis global que no es menos importante que la crisis ecológica, o la guerra, nos aleja del paradigma de que la educación de la población vuelve a la comunidad local o las grandes ciudades un mejor lugar para vivir y convivir con los demás. Ahora se vive la cultura de la sospecha de uno contra todos y todos contra uno. Se desconfía hasta de aquellos que están llamados a velar y proteger a la ciudadanía, de los representantes de las instituciones, del ciudadano que se acerca, todo como resultado del ambiente de inseguridad que vive la sociedad, cuya fuente y origen es la degradación humana, el ansia de tener que llevar al hedonismo y la impunidad. Pero sobre todo la pérdida del sentido de lo que es bueno y de lo que es malo, la supremacía del capital sobre el ser humano, la conversión de la persona como sujeto que tiene inteligencia, voluntad y libertad el cual debe ser el centro de toda acción humana y de las instituciones, para convertirlo más bien en objeto al cual se le puede categorizar en personas de primera, segunda o tercera categoría. Estamos ante una sociedad donde los medios opacan los fines, el mismo crecimiento económico se concibe como un fin en sí mismo y no como medio para el tránsito hacia el desarrollo. La tecnología se absolutiza y no se la usa como instrumento para ayudar a salir del circulo de la pobreza y la degradación ambiental, sino para acabar con todo bajo la óptica de que todo es reemplazable, sin caer a la cuenta de que la naturaleza tiene límite y es tan necesario cuidarla, conservarla y rehabilitarla para garantizar de que hayan bienes de consumo, trabajo y recursos naturales para las presentes y futuras generaciones que a la vez garantice su reproducción para garantizar la vida del ser humano que ejerce su acción para 
transformarla y así garantizar la existencia de la humanidad. Estamos ante el reto de reafirmarnos como sujeto, luchando por la vida, mediante la postulación de una ciencia accesible a todos, la búsqueda de la equidad y la inclusión y el cuidado de la naturaleza.

En una sociedad democrática, resultado de una educación democrática y con rigor científico no se puede impulsar diferentes tipos de educación, que darían lugar a la generación de diferente estructura curricular con diferentes contenidos programáticos, desigualdad en la instalación de infraestructura tecnológica, equipo, espacios físicos para la recreación y el deporte y sobre todo diferencia en la aceptabilidad en el mercado laboral o diferentes estándares de beneficios o rendimientos sociales. Una sociedad más igualitaria, demanda una educación igualitaria concebida como bien social en la que los distintos estratos sociales disfruten del arte, el deporte, la tecnología, las bibliotecas, el esparcimiento, la calidad y el rigor científico. Tanto en la ciudad como en el campo.

La educación es una inversión económica pero también es una inversión social que implica costos La educación no es un gasto la educación es una inversión, práctica social para el estímulo intelectual, el desarrollo de habilidades y conocimientos que inducen al incremento de la productividad, para beneficio de la gente, las empresas y la sociedad en general. Invertir en un bien de capital, en una instalación física es precisamente porque se espera tener un rendimiento monetario a corto o largo plazo, sin embargo, invertir en educación, aunque genera rendimientos monetarios, pero más importante es el rendimiento social o no monetario que produce. Estos rendimientos no palpables a la larga producen mayor satisfacción personal y social. Estos son los mejores rendimientos y quizá no se perciban porque no se reflejan directamente de manera visible, pero se reflejan en las externalidades o los efectos de la educación, que si se miden siempre resultara algún rendimiento monetario, tanto personal como social. Esta situación no siempre se comprende ni por los que planifican la inversión social, ni por los mismos académicos, que generalmente obvian el papel social de la educación, dándole primacía a la rentabilidad económica como resultado del ejercicio profesional de quien recibe la educación.

También es importante destacar que de toda inversión se espera una rentabilidad sea económica o social, pero al mismo tiempo genera costos. En el caso de la educación sea inversión o se conciba como gasto, es importante además de analizar la función económica de la educación, también es imprescindible la interpretación económica, en tanto que la educación como servicio estratégico en toda sociedad implica dilucidar quién es el que en realidad se responsabiliza por los costos y los gastos que “el saber" implica. Esta preocupación del análisis económico es lo que justifica que en la economía de la educación se dé prioridad al estudio de los rendimientos, tasa de retorno, costos y gastos, financiamiento público y financiamiento privado de la educación.

\section{Educación para el desarrollo y no solo para el crecimiento}

Hasta aquí se ha reflexionado de los efectos sociales e individuales de la educación en el proceso de desarrollo social. Sin embargo, es importante resaltar el hecho de que es en la educación donde se debe poner el acento, como elemento clave, para alcanzar un crecimiento económico sostenido, que lleve al aspecto cualitativo de desarrollo. Transmitir conocimiento es apostarle al desarrollo. Es a través de la educación que los aspectos cuantitativos del crecimiento, se convierten en aspectos cualitativos del desarrollo. A. Page 1977 "La educación torna más eficiente el trabajo, y con ello más productivo (P.15) Como la educación es un sector estratégico, corresponde al Estado asumir la mayor responsabilidad en la postulación de una política educativa para el "deber ser", que humanice y socialice y que tenga como horizonte el desarrollo económico y social que incluya a todos.

De nada serviría las nuevas tecnologías, si las poblaciones no las pudiesen asimilar por falta de una educación adecuada. No se puede alcanzar incremento de la productividad, como relación de eficiencia resultado del progreso tecnológico si no se cuenta con una fuerza laboral capacitada, educada 
y con capacidad de recibir instrucciones para responder positivamente al ritmo en que avanzan las comunicaciones, el cálculo rápido y el progreso técnico. Hay una relación directa entre avance técnico científico y educación de la población: a mayor progreso tecnológico acompañado de complejidad, debe haber mayor educación e información de la población, sobre todo de los trabajadores que manipularan y aplicaran la tecnología en las unidades económicas donde laboran. Los conceptos acuñados de inversión social, inversión humana, inversión en talento, inversión en capital humano justifican el estudio de la economía de la educación como disciplina para dilucidar otros conceptos relacionados con: las externalidades, rentabilidad de la educación, costo de la educación y la relación del nivel educativo con los sectores y actividad productiva.

A estos conceptos explicados explícitos o implícitamente vale la pena agregar otros conceptos propios de la economía de la educación relacionados con el capital humano de carácter inmaterial relativos a las capacidades, habilidades, destrezas y conocimientos no materiales de las personas. Que sumado el conocimiento y capacidades incorporados producto de la instrucción la reserva total de conocimientos lo califica como capital de capacitación. Estos últimos dos conceptos para caracterizar el ser humano implican una dimensión ética porque la persona no puede ser capital, en virtud de que el ser humano no se desprecia en tanto que el capital por su uso o antigüedad sufre un proceso de depreciación, por tal motivo el concepto introducido de capital humano es cuestionable y amerita un debate para hacer una reflexión más profunda al respecto.

Otro elemento a considerar es la decisión de los individuos de trabajar o estudiar. Si trabaja dejaría de percibir los beneficios de la educación que a más largo plazo le podría generar rendimientos monetarios, o estudiar razón por la cual a corto plazo dejaría de recibir un rendimiento monetario por estar estudiando y dejar de trabajar. Esto es el costo de oportunidad. Crecimiento económico elemento cuantitativo que aumenta la base material de la sociedad y puede inducir al desarrollo económico concebido como un elemento cualitativo que incluye los elementos cualitativos del crecimiento económico y el progreso técnico en favor de la mayoría de la gente.

Finalmente vale la pena enfatizar que la educación y la economía son ciencias ideográficas, no experimentales pero probabilísticas. Ciencias históricas con principios, leyes y modelos que se relacionan entre sí. J. Malta Luna 2002 "La relación ente educación y economía no es técnica" (P.1) en donde el hecho social o económico no es replicable, pero sus resultados se fundamentan en el método científico como método único, independientemente de los juicios de valor a los que es difícil escapar, y que permiten tener distintas percepciones de la realidad, percepciones que no pueden cambiar la verdad, como objeto propio del deseo de saber.

\section{Consideraciones finales}

1. La educación es un derecho, pero también es una inversión. Como derecho, debe ser prioridad en la agenda de los gobiernos y de la cooperación internacional. Evitando hacer de ella un medio para la máxima ganancia en función del interés privado.

2. La educación es un reflejo del modelo económico que se impulsa, la política educativa queda subordinada a la política económica y la actividad educativa se incorpora bajo la modalidad de las actividades simples y mecánicas que desarrolla un aparato productivo rezagado.

3. El concepto de capital humano encierra una dimensión ética, porque el capital se deprecia, por el uso y desgaste que sufre, ejemplo la maquina como bien de capital, se deprecia el dinero capital financiero. Entonces desde esta perspectiva del capital humano, el ser humano se deprecia cuando deja de ir a la escuela, cuando se envejece o cuando se le olvidan las cosas. A ese nivel de deshumanización se ha llegado. El ser humano no puede ser capital, el ser humano es ante todo persona que está dotado de inteligencia, voluntad y libertad. 
4. En una economía marcada por desequilibrio entre oferta laboral y demanda laboral, quienes tienen los medios de producción son los que tienen la opción de invertir y al haber sobreabundancia de personal no calificado y calificado en condición de desempleado, quien invierte juega con los salarios. Esto no solo genera desinterés por incorporar progreso técnico, porque prefiere contratar mano de obra abundante en desempleo para inclusive pagarla hasta por debajo del salario mínimo

5. Aunque el mito de que con solo educación se va a salir de la pobreza, se viene abajo o se desvanece en algunos países que, como Honduras, tienen mucho personal altamente calificado que vive en la pobreza, esto es así porque la mayoría de los empleadores que los invade el "miedo" a emprender procesos complejos y con ello a no emplear personal bien preparado profesionalmente, porque tendrían que ofrecerles mayor remuneración por su trabajo.

También es cierto que se encuentran casos eventuales de personas que obtienen altos rendimientos en las actividades que realizan, algunos de los cuales están dentro del sector informal de la economía, sin ser personas de mayor educación. 


\section{REFERENCIAS BIBLIOGRÁFICAS}

Boff, Leonardo. Cuidar la Tierra: hacia una ética universal. Ediciones Dabar, México, D.F. 2001

Dierckxsens, Wim. La crisis mundial del siglo XXI: oportunidad de transición al poscapitalismo. Ediciones desde abajo. Departamento ecuménico de investigación (DEI). Bolivia, 2009

Flores Silva, Jorge. Acerca del curriculum en la Universidad Nacional Autónoma de Honduras: elementos de discusión para una propuesta. Revista Economía Política $\mathrm{N}^{\circ} 30$. Facultad de Ciencia Económicas. Instituto de Investigaciones Económicas y Sociales IIES-UNAH. Honduras 2004

Hinkelammert, Franz (compilador). El huracán de la globalización. Editorial Departamento Ecuménico de Investigaciones (DEI). Costa Rica, 1999

Labarca, Guillermo (compilador). Economía Política de la educación. Editorial Nueva Imagen. México, 1980

Malta Luna, Juan Joseph. Economía de la Educación: gestión financiera de proyectos educativos. Impreso en Ideas Litográficas. Colección Gestión Educativa. Honduras, 2002

Page, André. La economía de la educación. Editorial Kapelusz. Colección estudios e investigaciones. Argentina, 1977. 